\title{
Reliability of magnitude matching
}

\author{
LAWRENCE E. MARKS \\ John B. Pierce Laboratory and Yale University, New Haven, Connecticut
}

\begin{abstract}
In each of four experimental sessions, each of 16 subjects gave magnitude estimates of the taste intensities of $\mathrm{NaCl}$ and the loudness of noise on a single, common scale-the method of magnitude matching. In all sessions, the intensity levels of the noises were identical; but in two sessions, the concentrations of $\mathrm{NaCl}$ were low, and in two they were high. Cross-modality matches (magnitude matches) between $\mathrm{NaCl}$ and noise were derived from the judgments, revealing two main findings: First, given constant $\mathrm{NaCl}$ concentrations, individual subjects showed reliably different magnitude matches. Second, changing the $\mathrm{NaCl}$ concentrations (context) strongly affected the magnitude matches. These findings suggest that magnitude matching may be useful in assessing interindividual as well as intergroup differences, though caution must be taken to minimize effects of context: Context effects are pervasive; they suggest the presence of a complex relativistic process operating when people judge the intensities of qualitatively different stimuli.
\end{abstract}

For the last several years, my colleagues and I have been examining the virtues-and the limitations-of the method of magnitude matching (Marks et al., 1988; Marks, Szczesiul, \& Ohlott, 1986; Stevens \& Marks, 1980). In magnitude matching, subjects try to judge the perceived intensities of stimuli from two different sensory modalities, $\mathrm{A}$ and $\mathrm{B}$, on a single, common scale of perceptual magnitude. The judgments make it possible to generate cross-modality matching functions-functions describing the stimulus intensities on A that match, perceptually, stimulus intensities on B. Often, the practical goal is to establish whether individuals or groups differ in the way in which they perceive stimuli on Modality B, given the assumption that they are equivalent with respect to the way in which they perceive stimuli on A.

Magnitude matching has proven successful in identifying differences both between groups and among individuals. For example, magnitude matching showed that groups of subjects with categorically high absolute taste thresholds for the bitter substance 6-n-propylthiouracil (PROP) were less responsive to suprathreshold sucrose than were groups with low thresholds for PROP (Gent \& Bartoshuk, 1983; Marks et al., 1988). Moreover, individual subjects with high or low threshold sensitivity to PROP generally gave correspondingly high or low magnitude matches to suprathreshold PROP (Marks et al., 1988).

\section{Magnitude Matching and \\ Individual Differences}

In this paper, I ask more generally, how well does the method of magnitude matching apply to individual subjects? Is it sufficiently reliable to uncover small individual

This research was supported by NIH Grant NS 16993. I thank Veronica Stone for her assistance in collecting and analyzing the data. Please direct correspondence to Lawrence E. Marks, John B. Pierce Laboratory, 290 Congress Avenue, New Haven, CT 06519. differences in the perceived magnitude of suprathreshold stimuli? To ask these questions is to ask about the stability of cross-modality comparisons. Differences among people in cross-modality matching functions may imply differences in perception on one or both of the modalities in question, though it may not always be possible to decide which one. For example, to match the loudness of a band of noise to the taste intensity of $0.1 \mathrm{M} \mathrm{NaCl}$, one person may require an SPL of $80 \mathrm{~dB}$, whereas another may require only $70 \mathrm{~dB}$. Perhaps $\mathrm{NaCl}$ tastes stronger to the first person; perhaps noises appear stronger to the second person; or perhaps the two differ in some other, more complex, way with respect to both taste and loudness perception. Regardless of which explanation is correct for these two hypothetical people, or for any other two individuals (or groups), an empirical difference between cross-modality matches or magnitude matches may entail some perceptual difference between the individuals.

As already indicated, Marks et al. (1988) showed that magnitude matching can uncover relatively large differences among individuals, when these presumably have a sensory basis. Still lacking is an assessment of magnitude matching's ability to uncover relatively modest individual differences (such differences may have a sensory basis or may derive from idiosyncracies in judgment). So far, though, no one has established the reliability of the method in individual subjects-although Marks et al. (1986) reported relatively poor intraindividual consistency when the same subjects were tested with the same pairs of modalities but different scaling procedures, or with different pairs of modalities but the same procedure. Hence I pose here a simple and basic question: When a given person is tested on repeated occasions with the same modalities and the same scaling procedure, does she or he produce the same magnitude matches? The issue of intraindividual repeatability is intimately connected to the issue of interindividual variation. The present experiment asks whether individual differences (interindividual variation) are 
greater in size-and if so, how much greater-than the "noise" in measurement (intraindividual variation).

In formulating the present study, indeed, in using the method of magnitude matching to compare perception in two sense modalities across different individuals or groups, I am using a model that is characterized by Figure 1. The model assumes that both modalities in question-here loudness and taste-have associated with them a common dimension of perceived intensity, and that, at least within a given experimental session, loudnesses and taste intensities can be put into one-to-one correspondence. For each SPL of a band of noise and for each concentration of $\mathrm{NaCl}$ there is a corresponding level of perceived intensity, which the subject presumably maps onto a set of numbers. In principle, when a given loudness equals a given taste intensity, the subject emits a given numerical response. The question posed here is, do different individuals align the two sensory continua in reliably different ways?

\section{Effects of Context on Magnitude Matches}

To answer this question, I could simply test several people on different days with the same magnitude-matching paradigm and compare the results. But it is more informative to combine this test of reliability with a test of "contextual sensitivity." In several studies, my colleagues and I have shown that magnitude matches are highly sensitive to the stimulus levels presented within the test session (Marks et al., 1988; Marks et al., 1986); that is, magnitude matches do not provide wholly "absolute", equivalences.

Ideally, of course, magnitude matches would do so: If a noise of $40 \mathrm{~dB}$ has a loudness that matches, absolutely, the taste intensity of $0.1 \mathrm{M} \mathrm{NaCl}$ (as in Figure 1), then $40-\mathrm{dB}$ noise and $0.1 \mathrm{M} \mathrm{NaCl}$ should always produce the same average response (magnitude estimate or rating-scale

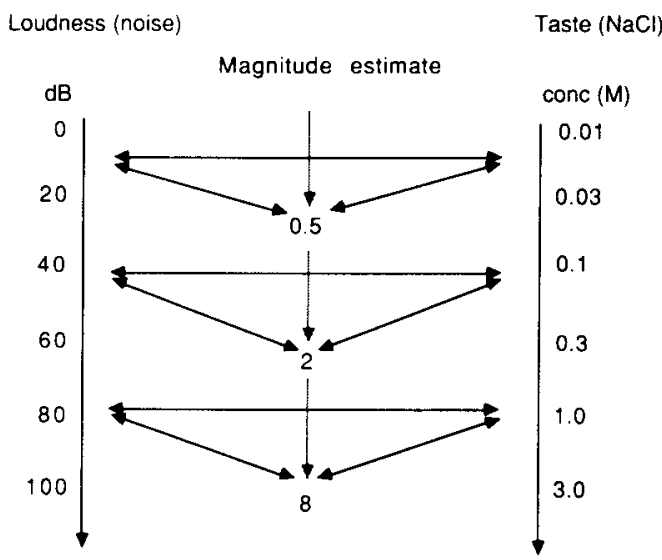

Figure 1. Model of magnitude matching: Sensation magnitudes on two perceptual modalities - here loudness and taste - are assumed to match, such that physical intensities on one modality align with physical intensities on the other. When magnitudes match, they produce, on the average, the same numerical response in magnitude estimation. judgment, depending on the scaling procedure), regardless of the particular levels of SPL or $\mathrm{NaCl}$ concentration presented in a session. But this is not so. Changing the mean intensity levels of the stimuli can change the cross-modality matches by a substantial amount; in log stimulus terms, the implicit cross-modal matches change by about $50 \%$ of the size of the shift in stimuli (Marks et al., 1988; Marks et al., 1986). If subjects judge loudness and taste, and the tastant concentrations are augmented by a factor of 10 ( $1 \mathrm{log}$ unit) across sessions, the concentration required to match a given sound level increases by a factor of about $3(0.5 \mathrm{log}$ unit). In terms of Figure 1, the two continua shift their alignment.

Note that this general rule-that contextual "bias" equals about $50 \%$ of contextual shift-characterizes the performance obtained by pooling data over subjects; little is known about how individuals respond to changes in stimulus context. People seem to vary widely in the amount of contextual effect they display in a particular experiment, some showing essentially absolute matching, and others wholly relative matching (Marks et al., 1986). But without measures of individual reliability, we still do not know whether such variation represents a stable characteristic of each person's scaling performance. In the present experiment, I therefore sought to answer two questions-one about individual reliability, the other about individual responses to changing stimulus context.

\section{METHOD}

\section{Stimuli}

The stimuli were five intensity levels of sound and eight concentrations of $\mathrm{NaCl}$, like those used by Marks et al. (1988, Experiment 6). The sounds were low-frequency $(100-400 \mathrm{~Hz})$ bands of flat-spectrum noise, produced by filtering (Allison 2B filter, $48 \mathrm{~dB} /$ octave rolloffs) the output of a Lafayette white-noise generator. The intensity of each sound-30, $44,58,72$, and $86 \mathrm{~dB}$ loudness level (LL) - was set by a General Radio decibel attenuator, the output from which fed a calibrated pair of TDH-39 headphones mounted in MX41/AR cushions. Loudness levels were determined by matching the loudness of each band of noise and 1000-Hz tone.

The taste stimuli consisted of reagent grade $\mathrm{NaCl}$, dissolved in deionized water to concentrations of 0.032 to $1.8 \mathrm{M}$ in $0.25-\mathrm{log}$ unit steps and presented at room temperature (approximately $21^{\circ} \mathrm{C}$ ) in plastic cups containing $5 \mathrm{ml}$ of solution. The eight concentrations were then subdivided into two subsets, one of low concentrations $(0.032-0.32 \mathrm{M})$ and one of high concentrations $(0.18-1.8 \mathrm{M})$. Note that the subsets overlapped, in that 0.18 and $0.32 \mathrm{M}$ were the two highest concentrations of the low subset and the two lowest concentrations of the high subset.

\section{Procedure}

The procedure was magnitude matching by means of magnitude estimation with no prescribed standard stimulus or numerical response modulus (Stevens \& Marks, 1980). The sounds and tastes were presented on alternate trials; otherwise, the stimuli were randomly ordered with regard to LL and concentration. On sound trials, the subject presented the noise for $1 \mathrm{sec}$ by depressing a hand-held switch. On taste trials, the subject sipped the contents of the cup, then expectorated, rinsing the mouth before and after each trial with distilled water to help minimize effects of adaptation. On each trial, the subject tried to assign a number to the perceived intensity of the stimulus, sound or taste. To the first stimulus, the subject was 
instructed to assign whatever number seemed best to correspond to its perceived intensity; then the subject was to assign numbers to other stimuli in proportion. The subjects were carefully instructed to judge both loudness and taste intensity on a common scale of magnitude. Thus, if a particular sound and a particular taste appeared equally strong, they were to receive the same number. The experimenter told the subject not to assume that the ranges of loudness and taste intensity would necessarily be the same; the sounds could appear stronger than the tastes, or the tastes stronger than the sounds.

Over the course of a given session, the entire ensemble of 10 stimuli (5 sounds and 5 tastes) was judged a total of five times. Sixteen young men and women served as subjects, each participating in four sessions, which were held on different days (2-10 days separating successive sessions). None had any evident or known defect of taste or hearing. For half of the subjects, the first session contained the low-concentration series of tastes (L); for half, it contained the high-concentration series $(H)$. The sessions then alternated between low and high, so that half of the subjects followed the order $\mathrm{L}_{1}-\mathrm{H}_{1}-\mathrm{L}_{2}-\mathrm{H}_{2}$ and half the order $\mathrm{H}_{1}-\mathrm{L}_{1}-\mathrm{H}_{2}-\mathrm{L}_{2}$. Thus each subject served twice in both the low- and the high-concentration conditions, thereby permitting an assessment of individual reliability under each contextual condition.

\section{RESULTS}

\section{Group Analysis}

To provide a framework for analyzing the results of individual subjects, I first examined group results by pooling the data in each condition, arithmetically within subjects and geometrically across subjects. The means appear in Figure 2 (upper four panels for the subjects who received the conditions in the order $\mathrm{L}_{1}-\mathrm{H}_{1}-\mathrm{L}_{2}-\mathrm{H}_{2}$, lower four panels for the subjects who received $\mathrm{H}_{1}-\mathrm{L}_{1}-\mathrm{H}_{2}-\mathrm{L}_{2}$ ). In each panel, the estimates of taste intensity (open squares) and loudness (filled squares) are plotted against their respective stimulus magnitudes.

The most striking feature of the results is the substantial effect of context. This effect shows itself as a shift in values of implicit cross-modality matches (magnitude matches). Recall that two of the concentrations of $\mathrm{NaCl}$, 0.18 and 0.32 , were common to the low and high series, falling at the top end of the one series and at the bottom of the other. Consider the magnitude matches between, for example, $0.18 \mathrm{M} \mathrm{NaCl}$ and $\mathrm{LL}$ of noise. We assess these matches by determining, via linear interpolation on the functions of Figure 2, the $\mathrm{LL}$ and the $\mathrm{NaCl}$ that produce the same magnitude estimate. Thus in the first low-concentration series $\left(L_{1}\right)$ given to subjects who received the order $\mathrm{L}_{1}-\mathrm{H}_{1}-\mathrm{L}_{2}-\mathrm{H}_{2}$ (upper left-hand panel), $0.18 \mathrm{M} \mathrm{NaCl}$ received an average magnitude estimate of 23.6 , slightly greater than the average estimate of 22.0 given to noise of $72 \mathrm{~dB}$ LL. By interpolation, $0.18 \mathrm{M}$ $\mathrm{NaCl}$ matched $73.4 \mathrm{~dB}$ (determined by interpolation on the loudness function). In that group's first highconcentration series, $\mathrm{H}_{1}$ (upper right-hand panel), $0.18 \mathrm{M}$ $\mathrm{NaCl}$ received a much smaller magnitude estimate, 6.64 , interpolated on the corresponding loudness function to match an LL of $56.2 \mathrm{~dB}$. The two cross-modality matches, obtained with different sets of $\mathrm{NaCl}$ concentrations, diverge, therefore, by a substantial $17 \mathrm{~dB}$.
Comparable shifts in magnitude matches are evident at all levels of $\mathrm{NaCl}$ concentration, and in the data of both groups of subjects. Indeed, a second feature of the average results is the uniformity of the context effects, both over repetitions within each group and across groups receiving different orders of conditions.

To analyze these effects, I first calculated, for each subject and each condition, the LLs that matched $0.18 \mathrm{M}$ and $0.32 \mathrm{M} \mathrm{NaCl}$; I then summarized each subject's performance in each session with the average of the two LLs. ${ }^{1}$ An analysis of variance (ANOVA) conducted with one between-subjects variable (order of contextual conditions) and two within-subject variables (contextual condition and replicate) showed only the effect of context to be reliable $[F(1,14)=47.98, p<.0001]$. Order did not matter $[F(1,14)=2.378, p=.15]$; neither did replicate $[F(1,14)$ $=1.289, p=.28],{ }^{2}$ nor did any of the interaction terms (all values of $F<1.0$ ). Given that order was insignificant, it is possible to ignore this variable in the analysis of individual differences, which constitute the primary focus of this study, as described below.

\section{Individual Differences: \\ Magnitude Matches and Context Effects}

Table 1 lists the individual "matching points." Perusal of the table is instructive, in that it can help answer the questions posed in the introduction.

First, with repetition of the same stimulus conditions, how consistent are individuals? Despite the substantial variability from day to day in several of the subjects' magnitude matches under the same conditions (intraindividual variation), the differences across individuals (interindividual variation) are even greater. Subject 5 , for instance, gave an average matching level of $85 \mathrm{~dB}$ in the low- $\mathrm{NaCl}$ condition, whereas Subject 13 gave an average of $60 \mathrm{~dB}$ in the same condition. Statistically, the consistency of individuals is supported by the results of a second ANOVA (conducted now with the two main variables of subjects and contextual condition, the interactions with replicates providing error terms), showing a reliable effect of subjects $[F(15,15)=8.509, p<.001]$. Thus quantitative analysis indicates that, overall, the differences in magnitude matches across individuals greatly exceed the fluctuations within individuals. The individuals were indeed consistent across similar testing conditions.

What about individual consistency or variation in the size of the contextual shifts? Table 1 reveals that virtually every subject shows such a shift; the matches to $0.18 \mathrm{M}$ and $0.32 \mathrm{M} \mathrm{NaCl}$ averaged $74 \mathrm{~dB}$ in the low- $\mathrm{NaCl}$ conditions, but only $55 \mathrm{~dB}$ in the high- $\mathrm{NaCl}$ conditions, consistent with the group analysis described above. But there is no statistical evidence that individual subjects differed reliably in the magnitude of these contextual shifts. Although some subjects did show large contextual shifts, and other subjects small ones, these effects also tended to vary considerably across replicates; the interaction of subject $\times$ context is not reliable $[F(15,15)=$ $1.863, p=.13]$. By and large, then, the subjects did 
Loudness Level (dB)
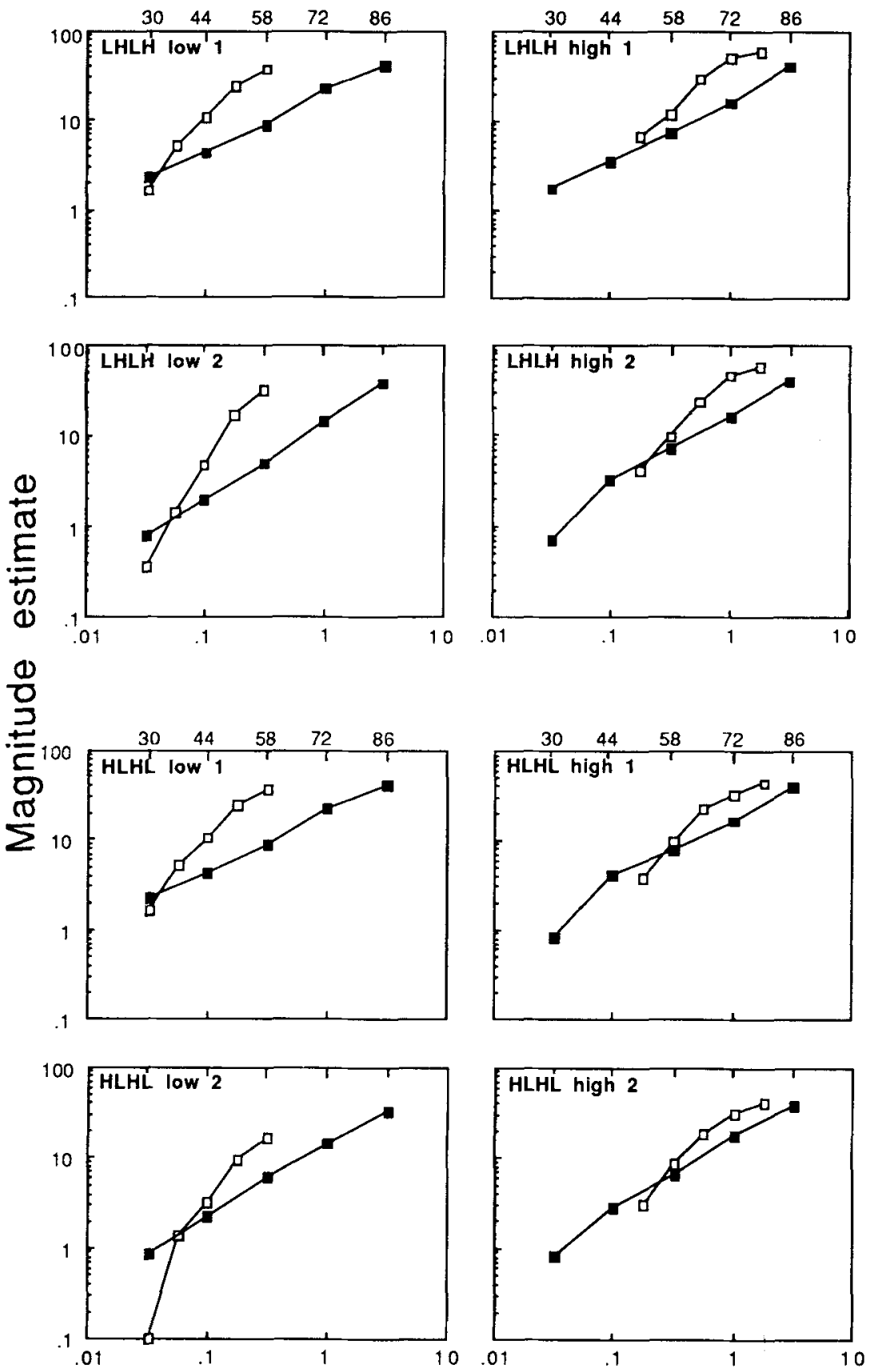

Concentration $\mathrm{NaCl}(\mathrm{M})$

Figure 2. Average magnitude estimates given to the taste intensity of $\mathrm{NaCl}$ (open squares), as a function of molar concentration (lower abscissa), and to the loudness of bands of lowfrequency noise (filled squares), as a function of loudness level in decibels (upper abscissa). In any given session, the concentrations of $\mathrm{NaCl}$ were either low $(L)$ or high $(\mathbf{H})$ : The upper four panels and the lower four panels give results for the subjects who participated in the four sessions in the orders $L_{1}-H_{1}-L_{2}-H_{2}$ and $H_{1}-L_{1}-H_{2}-L_{2}$, respectively. 
Table 1

Magnitude Matches of Loudness of Noise (dB LL) to Taste Intensity (Mean of Matches to 0.18 and $0.32 \mathrm{M} \mathrm{NaCl}$ )

\begin{tabular}{cccccc}
\hline & \multicolumn{2}{c}{ Low NaCl } & & \multicolumn{2}{c}{ High NaCl } \\
\cline { 2 - 3 } \cline { 5 - 5 } Subject & Session I & Session 2 & & Session 1 & Session 2 \\
\hline 1 & 78.1 & 84.7 & & 78.0 & 54.3 \\
2 & 72.6 & 73.6 & & 51.7 & 44.0 \\
3 & 80.0 & 81.4 & & 66.2 & 55.6 \\
4 & 88.9 & 71.1 & & 31.2 & 42.0 \\
5 & 87.2 & 82.6 & & 64.4 & 67.5 \\
6 & 77.1 & 75.8 & & 40.9 & 50.5 \\
7 & 72.8 & 72.8 & & 56.7 & 63.1 \\
8 & 75.9 & 74.4 & & 84.7 & 68.9 \\
9 & 74.5 & 71.0 & & 64.9 & 38.2 \\
10 & 75.9 & 76.3 & & 63.2 & 67.5 \\
11 & 83.1 & 76.6 & & 55.0 & 65.3 \\
12 & 76.0 & 74.4 & & 58.8 & 58.1 \\
13 & 58.1 & 61.6 & & 47.2 & 50.2 \\
14 & 67.9 & 68.9 & 65.3 & 61.6 \\
15 & 65.1 & 72.1 & & 44.8 & 47.7 \\
16 & 65.6 & 57.6 & 26.8 & 38.7 \\
Mean & 74.9 & 73.4 & 56.2 & 54.6 \\
\hline
\end{tabular}

réspond similarly to changing context. Any apparent interindividual variation in contextuality can be chalked up mostly to intraindividual variation - that is, to noise.

\section{DISCUSSION}

\section{Practical Implications: Evaluating Individuals}

This dual finding - consistent (reliable) individual differences in levels of magnitude matches, plus uniformity in the way in which stimulus context affects the matching values-has an important practical implication: It suggests that, as long as appropriate precautions are taken, magnitude matching may prove useful in the evaluation of interindividual as well as intergroup differences in suprathreshold perception. The present results indicate clear individual consistency when the same modalities are compared with the same scaling procedure, complementing findings such as those of Collins and Gescheider (1989), who reported that power-function exponents calculated from absolute magnitude estimates were consistent with exponents derived directly from cross-modality matches.

In clinical as well as experimental settings, it is often desirable to assess suprathreshold sensory functioning. Until now, however, it has not been established how well the method of magnitude matching might serve to evaluate individuals. The present results indicate that individual magnitude-matching functions are reasonably robust, and that reliable individual differences evidence themselves even within nonclinical populations.

Moreover, the evidence that individuals respond more or less uniformly to changing stimulus context implies that, in principle, one could use iterative testing to establish more and more precise magnitude matches. From an initial magnitude-matching experiment one can choose matching stimulus levels to use in a subsequent experiment, the goal of iteration being to equate more closely the overall range and levels of subjective intensities on the two modalities. If, for example, an initial experiment shows Subject 1 giving greater responses to taste than to loudness and Subject 2 greater responses to loudness than to taste, in a subsequent experiment the sound levels can be raised for Subject 1, and the taste concentrations for Subject 2; that is, the stimulus intensities are set to levels that would have matched cross-modally in the previous experiment. The use of such an iterative procedure itself relies on the assumption that contextual effects are minimal when the two sets of subjective levels-tastes and loudnesses, for example-are identical. Marks et al. (1988, Experiment 10) used this very procedure with groups of young and old subjects to obtain more accurate measures of differences in suprathreshold odor sensation-more accurate, that is, than measures obtained when both groups received the same sets of stimuli. Encouraged by the present results, I infer that such iterative testing can improve measures on individuals as well.

\section{Theoretical Implications}

Individual differences. The present findings also bear two important theoretical implications. One concerns individual differences in psychophysical scales. Some subjects have commented, at the end of a test session in which they made magnitude matches of taste and loudness, that the taste stimuli produced much the stronger sensations, whereas others have commented that the sounds were stronger. The present results are consistent with such comments, suggesting thereby the possibility of real individual differences in sensory magnitudes. To be sure, it would not be possible to tell whether Subject 1, who matches relatively high decibel levels to tastes, has greater suprathreshold taste sensations, or weaker loudness sensations, than Subject 2, who matches relatively low decibel levels to the same tastes. Regardless of which sensory system differs-and it is quite likely, if substantial interindividual fluctuations do exist, that they occur in all sensory modalities-the very existence of such differences may require modifications in theories of psychophysical magnitude that postulate a uniform range of sensory response across all modalities ( $R$. Teghtsoonian, 1971). If it is appropriate to extrapolate the present findings to sensory judgments at the top end of the stimulus range-that is, to levels of "maximum" sensation-then, even in people with "normal" sensory systems, maximal sensory magnitudes may vary importantly from one modality or another, as well as in different ways from person to person.

Note that I have couched these conclusions as tentative. An alternative to the presumption of real sensory differences among people is the hypothesis that the individual differences are circumstantial; that is, they may arise because people enter the experimental setting with different past exposures, experiences, and expectations, in short, with idiosyncratic prior histories; presumably, these differences in prior history could lead, through different patterns of judgmental "bias," to differences in magnitude 
matches. By this account, the differences in magnitude matches are unrelated to any interindividual differences in underlying magnitudes of the sensory experiences. In order to explain the consistency of magnitude matches across sessions, however, it would also be necessary to postulate that subjects tend to remember, from one session to another, something about the judgments given to the stimuli in prior sessions. Ward (1987) has documented effects of memory on judgments made on different days, and $M$. Teghtsoonian and R. Teghtsoonian (1983) found transient consistency in individual exponents of crossmodality matches, which they related to memory.

In a fundamental sense, the two hypotheses just outlined provide two different accounts of the "absolute" component to magnitude matching. These accounts resemble, though they are not identical to, Ward's (1987) distinction between "absolute 1 " and "absolute ${ }_{2}$ " mappings of stimuli (or sensations) and responses. Absolute 1 mappings refer to hypothesized absolute connections between perceptual stimuli and the subjects' numerical responses (e.g., magnitude estimates); Zwislocki (1983; Zwislocki \& Goodman, 1980) has been a primary and articulate proponent of the view that such absolute connections do exist, revealing themselves most clearly when subjects are appropriately instructed freely to match number magnitudes to sensation magnitudes. Absolute 2 mappings refer to the particular mappings between stimuli and responses that subjects make in a given experimental situation; such mappings may change with changes in stimulus range, instructions, and so forth. Absolute $e_{2}$ mappings need not entail absolute $_{1}$ mappings, though absolute ${ }_{1}$ mappings do entail absolute ${ }_{2}$ mappings.

If absolute ${ }_{1}$ mappings between sensations and responses do exist, we would have a ready explanation of reliability in magnitude matches: Loudness $A_{L}$ maps absolutely onto Response R; Taste Intensity $A_{T}$ maps absolutely onto Response $R$; thus $A_{L}$ matches $A_{r}$. But the presence of pervasive context effects imply that there was considerable "looseness"' to the couplings between modalities.

Central to the second explanation of the present results is the role of memory. How might memory for performance in one session affect performance in a subsequent session? Most people make few, if any, overt quantitative judgments of sensory magnitudes during everyday situations, from which the present experimental setting clearly differentiates itself. Thus subjects may find it relatively easy to remember at least the range of their numerical responses.

What role might such memories play? Perhaps the subjects remember not only their specific responses or range of responses, but also the perceptual magnitude associated with each response, and then they tend to repeat the responses when the magnitudes recur. Were this so, we would expect a high degree of test-retest reliability, even without the presence of "real," sensory-based individual differences in perception: Even if each subject's magni- tude matches in the very first session simply reflected idiosyncratic or other nonperceptual choices of the moment, as long as the subject remembered the specific stimulusresponse connections, similar magnitude matches would obtain in subsequent sessions.

But if the subjects did remember specific connections between particular sensory events and particular responses (Ward's, 1987, absolute 2 mapping), then we would also expect individual subjects to show a high level of reliability across sessions in which the stimulus levels changed. But this was not the case. Magnitude matches remained unchanged only when the stimulus levels were the same, changing dramatically when the stimulus levels (context) shifted. This sensitivity to context shows that if memory does play a prominent role, what is remembered is not a set of absolute connections between stimuli and responses.

A second possibility is that the subjects remember something about the responses that they made in the prior session, perhaps the lowest and highest numbers given, but not the associated perceptual magnitudes. In this case, idiosyncratic choices of responses, subsequently well remembered, could lead to both high intraindividual reliability when the same stimulus levels are presented in different sessions and a high level of contextuality (relative judgment) when different stimulus levels are presented in different sessions. This account may also explain why Marks et al. (1986) found little individual consistency when different scaling procedures or pairs of modalities were tested in different sessions.

Context and magnitude matches. Finally, the present results bear on the still-unanswered question of why stimulus context has so potent an effect on magnitude matches. Conceivably, the effect could be due in part to differential, residual effects of adaptation on the taste perceptsthough the water rinses and the alternation of taste with noise were aimed at minimizing any possible effects of adaptation. In general, it is clear that people cannot fully abstract sensory magnitudes from qualitatively different experiences and judge these magnitudes on a single, common scale. Instead, judgments of magnitude represent a compromise between two components-an absolute component, which presumably depends on the underlying sensory events, and a relative component, which depends on the particular stimulus levels recently presented for judgment.

What processes are responsible for contextuality? Marks (1988) found context-dependent shifts in within-modality judgments (loudness of tones that differ in frequency) like those found in cross-modality comparisons. Several years ago, my colleagues and I (Marks et al., 1986) suggested that short-term sequential dependencies might account in part for context-based shifts in cross-modality matches. This account was predicated on a particular characteristic of the magnitude-matching procedure: The qualitatively different stimuli are presented in strict alternation. Un- 
fortunately, the account predicts that contextual effects should diminish markedly when the stimuli are presented at random rather than in alternation; but this prediction was not confirmed (Marks, 1988).

An important clue to the source of contextual effects is the finding that the magnitude of the contextual shift depends quantitatively on the similarity between the stimuli being compared: For example, changing contextual intensity levels has a substantial effect on loudness matches derived from magnitude estimates of tones that differ widely in sound frequency (e.g., 500 and $2500 \mathrm{~Hz}$, in different critical bands), but it has virtually no effect on matches derived from tones that have similar frequencies (e.g., 1100 and $1130 \mathrm{~Hz}$, in the same critical band) (Marks \& Warner, 1990). This finding suggests that contextual interactions display stimulus selectivity. That is, the contextual process must have a built-in "filter," so that matching values stay matched when subjects judge qualitatively similar stimuli, but shift when subjects judge qualitatively different stimuli. It is interesting that Ward (1990) recently showed that sequential dependencies in magnitude judgments of loudness depend in part on whether successive stimuli fall in the same or in different critical bands.

Given that contextual shifts depend on "critical bands," they may derive from the operation of an attentional mechanism, like the "attention band" proposed by Luce and Green (1978)-a central mechanism that may be thought of as monitoring accurately the information deriving from only a small fraction of fibers (say, over a narrow range of sound frequencies), and less accurately all other information. If the location of the attention band stays relatively fixed over the course of the session-as implied by Marks's (1988) finding that the size of the contextual shift in loudness matches was the same when frequencies were alternated and when they were presented randomly-then only when all of the signals fall within a "critical bandwidth" can comparisons of magnitude be made on an absolute basis, and contextual shifts be avoided. When stimuli fall in different "critical bands" (widely separated frequencies or different modalities), one set of signals or both must be monitored with poor accuracy, permitting a relativistic component of judgment to come into play. Interestingly, this account leaves open the possibility that people can make absolute scaling judgments, per Zwislocki (1983), but implies that this will be possible only when all stimuli fall within a single critical band of attention.

\section{REFERENCES}

Collins, A. A., Gescheider, G. A. (1989). The measurement of loudness in individual children and adults by absolute magnitude estimation and cross-modality matching. Joumal of the Acoustical Society of America, 85, 2012-2021.

GeNt, J. F., BARToshUK, L. M. (1983). Sweetness of sucrose, neohesperidin dihydrochalcone and saccharin is related to the genetic ability to taste the bitter substance 6-n-propylthiouracil. Chemical Senses, 7, 265-272.

LUCE, R. D., GREEN, D. M. (1978). Two tests of a neural attention hypothesis for auditory psychophysics. Perception \& Psychophysics, 23, 363-371.

MArks, L. E. (1988). Magnitude estimation and sensory matching. Perception \& Psychophysics, 43, 511-525.

Marks, L. E., Stevens, J. C., Bartoshuk, L. M., Gent, J. F., RifKIN, B., STONE, V. K. (1988). Magnitude-matching: The measurement of taste and smell. Chemical Senses, 13, 63-87.

Marks, L. E., Szczesiul, R., \& OHLotT, P. (1986). On the crossmodal perception of intensity. Journal of Experimental Psychology: Human Perception \& Performance, 12, 517-534.

MARKs, L. E., WARNER, E. (1990). Magnitude estimation: The slippery context effect and critical bands. Manuscript submitted for publication.

Stevens, J. C., Marks, L. E. (1980). Cross-modality matching functions generated by magnitude estimation. Perception \& Psychophysics, 27, 379-389.

Teghtsoonian, M., Teghtsoonian, R. (1983). Consistency of individual exponents in cross-modal matching. Perception \& Psychophysics, 33, 203-214.

Teghtsoonian, R. (1971). On the exponents in Stevens' law and the constant in Ekman's law. Psychological Review, 78, 71-80.

WARD, L. M. (1987). Remembrance of sounds past: Memory and psychophysical scaling. Joumal of Experimental Psychology: Human Perception \& Performance, 13, 216-227.

WARD, L. M. (1990). Critical bands and mixed-frequency scaling: Sequential dependencies, equal-loudness contours, and power function exponents. Perception \& Psychophysics, 47, 551-562.

ZWISLOCKI, J. J. (1983). Group and individual relations between sensation magnitudes and their numerical estimates. Perception \& Psychophysics, 33, 460-468.

Zwislocki, J. J., Goodman, D. A. (1980). Absolute scaling of sensory magnitudes: A validation. Perception \& Psychophysics, 28, 28-38.

\section{NOTES}

1. The method for normalization is essentially the same as that used by Marks et al. (1986), although the present method requires fewer steps. Both methods ultimately rest on the shift in implicit cross-modality matches as defined by the subsets of stimuli in the different contextual conditions that produce an overlapping range of numerical responses.

2. Although magnitude matches did not differ across replicates, visual inspection of Figure 2 suggests some small changes in the form of the psychophysical function for taste. I shall simply note that in the low$\mathrm{NaCl}$ conditions, the taste functions at the lowest concentrations do bend more steeply in the second than in the first runs.

(Manuscript received October 2, 1989; revision accepted for publication July 24,1990 .) 